\title{
Stochastic modeling uncovers a novel mechanism underlying the evolution of mutation rates in sexually reproducing populations
}

\author{
Andrii Rozhok ${ }^{1}$, Niles Eldredge ${ }^{2}$, James DeGregori ${ }^{1,3}$ \\ ${ }^{1}$ Department of Biochemistry and Molecular Genetics, University of Colorado Anschutz Medical \\ Campus, Aurora, CO, USA \\ ${ }^{2}$ Division of Paleontology, American Museum of Natural History, New York, USA \\ ${ }^{3}$ University of Colorado Comprehensive Cancer Center, University of Colorado Anschutz Medical \\ Campus, Aurora, CO, USA \\ Correspondence to Andrii Rozhok (andrii.rozhok@cuanschutz.edu).
}

\begin{abstract}
Natural selection is believed to universally work to lower mutation rates (MR) due to the negative impact of mutations on individual fitness. Mutator alleles have only been found to be co-selected by genetic linkage with adaptive alleles in prokaryotes. Sexual reproduction substantially reduces genetic linkage, allowing selection to effectively eradicate mutator alleles. The current understanding, therefore, is that in sexually reproducing populations selection always works to lower MR, limited by the effective population size that determines the overall selection efficiency. In the present paper, we apply a Monte Carlo model of a sexually reproducing population and demonstrate that selection acting on MR does not universally favor lower MR but depends on the mode of selection acting on adaptive phenotypic traits. We demonstrate a unique previously unreported co-selective process that can drive the evolution of higher MR in sexually reproducing populations. Our results show that MR evolution is significantly influenced by multigenic inheritance of both MR and adaptive traits that are under selection. Our results also show that, contrary to the generally accepted axiom, population size appears not to affect the strength of selection uniformly but likely forms an intra-population gradient that generates a "biased sampling" process that has an opposite effect on selection strength and thus modulates or even negates the effect of population size on MR evolution. Based on our results, we propose an expanded population genetics theory of the evolution of mutation rates in sexually reproducing organisms. Our results have potential implications for understanding processes underlying rapid adaptive change in speciation and related macroevolutionary patterns.
\end{abstract}




\section{Introduction}

Mutation rates range widely among different species across the phylogenetic tree of life (1). A number of factors have been proposed to explain the MR diversity across taxa, such as genome size (2), variation in the trade-off between the cost of efficient DNA repair and speed of replication among taxa $(3,4)$, generation time $(5,6)$, metabolic rate $(6)$, and population size affecting the efficiency of selection in lowering MR (1). Many of the proposed mechanisms are based on evidence limited to certain taxonomic groups and theoretical speculation, therefore it is unclear presently how much they can be generalized across the tree of life. However, Lynch (1) does report a plausible inverse correlation between MRs and population sizes across a vast span of taxa, ranging from prokaryotes to mammals. Small populations appear to demonstrate higher $M R$, supposedly because of their lower responsiveness to selection and thus an increased inefficiency of selection in lowering $\operatorname{MR}(1,7,8)$.

Regardless of the factors involved, the overarching currently accepted presumption is that selection universally works to lower MR because of the negative effect of mutations on individual fitness $(1,9-12)$. Exceptions to this rule have been reported for asexually reproducing species whereby strong genetic linkage between mutator alleles and alleles important in evolutionary adaptation in some cases leads to co-selection for mutator alleles and thus a higher MR - the socalled "mutator hitchhiking" model (13-15). In sexually reproducing populations, however, genetic linkage is substantially reduced by the recombination process, and such mutator hitchhiking is deemed to be the exception, not the rule. However, genetic hitchhiking might not be the only mechanism that contradicts the presumption that MRs are universally lowered by selection. The following reasoning can be proposed to argue that the sign of selection acting on MR may likely be reversible even in sexually reproducing organisms.

Quantitative phenotypic traits with multigenic inheritance are often distributed in populations in terms of their expression, in a typical case following a normal distribution as shown in Fig. 1. Under stabilizing selection, the population's mean expression of the selected trait will tend to confer the highest fitness and thus be the most frequent (although deviations from normality do occur). Lower fitness phenotypes deviate toward the tails of the phenotypic distribution and occur at lower frequencies. Based on the multigenic inheritance of MRs and some emergent evidence (16-19), we can assume that MR is also a distributed trait in sexually reproducing populations. Many different genes including those involved in DNA replication, repair, damage avoidance and metabolism will impact the final $\operatorname{MR}(16,18)$. Such a highly polygenic inheritance implies that MR should be a distributed trait in populations that varies among individuals, and evidence from multiple studies already supports this view $(19,20)$. Hypothetically, individuals that are phenotypically closer to the population phenotypic mean should come from parents that have lower MRs and inherit, among the multiple genes encoding $\mathrm{MR}$, a collection of alleles that result in a relatively low MR. Correspondingly, individuals who inherit more alleles promoting higher MR should have higher chances of deviating from the mean and ending up in one of the tails of the phenotypic distribution. It is thus valid to assume that phenotypically more deviant individuals will tend to harbor alleles promoting higher $\mathrm{MR}$, and less deviant individuals will concentrate more alleles ensuring lower MR (Fig. 1). It follows then that stabilizing selection acting on the phenotype should increase the frequency of low MR alleles and decrease the frequency of high MR alleles in the population. However, when selection acting on 
the phenotype changes to directional (Fig.1), whereby tail phenotypes are favored, higher MR alleles should undergo enrichment and low MR alleles should decrease in frequency.

Based on the reasoning just laid out, it can therefore be hypothesized that, contrary to the current conventional presumption, the mode of selection acting on MR in sexually reproducing populations should not always lead to lower MR but should instead depend on the general selection regimen acting on adaptive phenotypic traits.

In the present study, we apply a Monte Carlo model faithfully replicating a sexually reproducing population of organisms to test this hypothesis and look deeper into factors that are critical in the evolution of MR. Monte Carlo modeling has been extensively used in many areas of science as a tool specifically designed to recapitulate in silico complex natural systems involving multiple independent processes. The complexity of such systems, notably including many biological processes, at times achieves levels that are not tractable by traditional analytical modeling, and analytical solutions for such processes often do not exist. This problem is particularly apparent in stochastic processes, such as genetic change and many aspects of sexual reproduction. Experimental approaches also are limited in their power to describe such systems due to the narrow focus of experimental manipulations, usually addressing only particular aspects of a complex system at a time. Monte Carlo modeling, therefore, is part of a very limited set of tools that can provide insights into how complex systems operate as a whole.

Our results reveal a previously unreported mechanism modulating the sign of selection acting on MR that is consistent with our hypothesis shown in Fig. 1. Our results demonstrate theoretical evidence that selection for higher $M R$ is possible and may be common in sexually reproducing organisms.

\section{A. Large population}

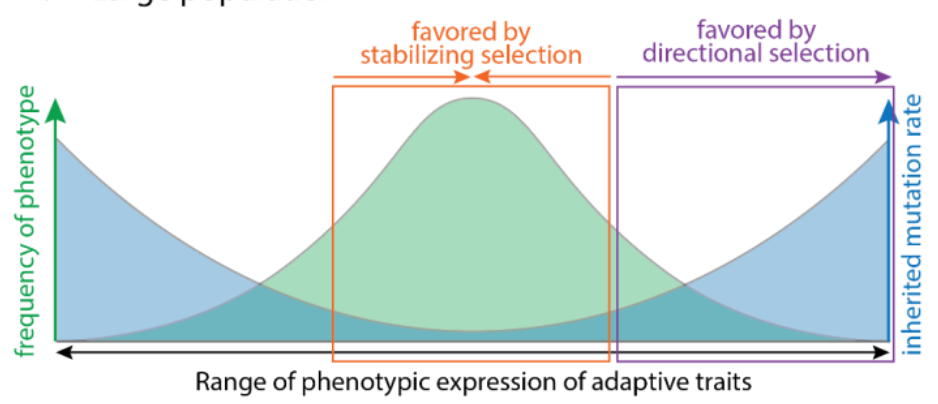

B. Small population

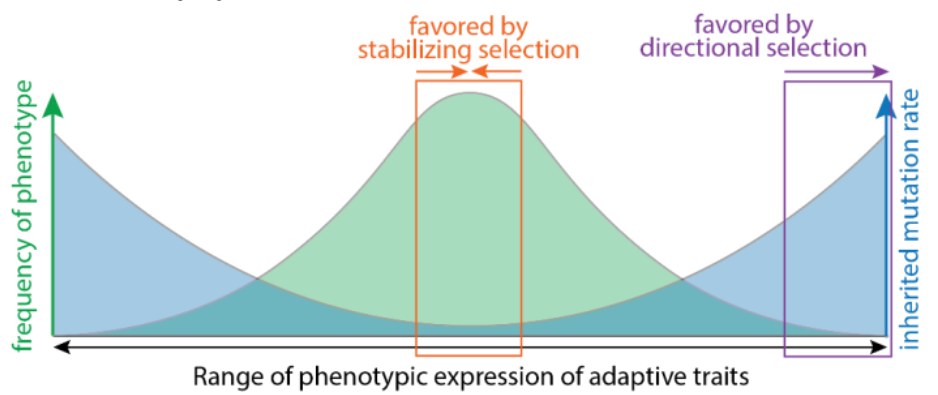

Fig. 1. A model for how selection regimen impacts the evolution of mutation rate in sexual populations. A, under stabilizing selection, the most adaptive phenotypes are close to the population mean/median; such phenotypes are more likely to be produced by parents with low germline MR in a population in which MR is a 
multigenic distributed trait. Under positive selection, the most adaptive phenotypes demonstrate unidirectional deviation of the selected trait(s) from the population mean. Such phenotypes are more likely to be produced by parents having higher germline MR and thus harboring multiple alleles conducive to higher MR; B, small population size reduces the strength of selection by increasing the strength of drift; this condition requires a phenotype to deviate sufficiently far from the population mean/median towards the selected tail to be responsive to selection. Such extremely deviant phenotypes in small populations are likely to come from parents with the highest germline MR and thus harboring fewer alleles for low MR. This condition should impede segregation of mutator alleles from adaptive alleles and introduce a selection bias toward the most extremely deviating phenotypes - we propose that this phenomenon opposes the effects of small population size and rescues population size induced reduction in selection efficiency.

\section{Methods}

The general model algorithm is explained in Fig. 2 and the code is provided in Supplement 1. All modeling was performed in Matlab (MathWorks Inc., Massachusetts). The model is a stochastic Monte Carlo discrete-event simulation that operates with an initial set of variables that change their state over time in discrete steps.

All simulation runs include 10,000 simulation steps. Under the standard set of parameters, the simulation is initially populated with 10,000 simulated individuals each having a genome consisting of two homologous chromosomes, each chromosome being inherited from respective parents. Each chromosome contains 10 genes that determine the mutation rate and 10 genes that encode a distributed quantitative adaptive trait. Each gene is assigned an initial value of "expression" that essentially determines the effect of the gene on the final phenotype. The average value for both MR and adaptive trait gene expression is 10 for simplicity. Each gene of any particular individual in the population is initially assigned an expression value $E$ equal to a

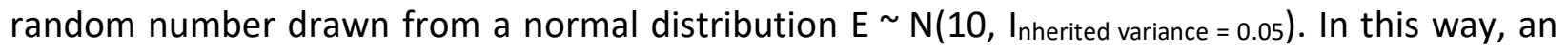
initial population's genetic variation is created. An individual's MR is then calculated as the average of the $E$ values of all MR-related genes. Similarly, the net phenotypic expression of an individual's adaptive trait is the average of the E-values of all genes that encode the trait. Therefore, both MR and the simulated adaptive trait are multigenic, with each gene equally contributing to the net phenotype for simplicity (i.e., none of the genes is dominant or negligible in determining the net phenotype relative to others). As a result of this setup, an initial population of 10,000 individuals is established that demonstrates a normally distributed variation of individual MR and adaptive phenotype, following a simple Shelford's law assumption (21).

At each simulation step $i$, several variable states are updated for each individual. First of all, each gene is tried in a binomial trial $M \sim B\left(1, C_{\text {urrent individual } M R}\right)$ for the possibility of a functional mutation that alters the gene's $E$-value. Genes whose resulting $M$-values equal 1 "mutate" by updating their $E$-value to $\mathrm{E}_{\mathrm{i}} \sim \mathrm{N}\left(\mathrm{E}_{\mathrm{i}-1}, I_{\text {nherited variance }}{ }^{*} \mathrm{C}_{\text {urrent individual } \mathrm{MR}}\right)$. In this way, at each step $i$ of the simulation, has a chance to obtain a functional mutation at an individual frequency $F_{j i}=M R_{j i}$, where $\mathrm{MR}_{\mathrm{ji}}$ current individual (j) mutation rate at step $i$. Mutant genes alter their current $E$-values with variance proportional to the species-specific inherited variance parameter (which is always set to 0.05) and the current individual mutation rate.

At each step $i$ of the simulation, each gene may recombine by swapping chromosomes with its homolog at the frequency 0.05 , which is set as a species-specific trait that does not vary individually. Genetic recombination probability for each gene awas established in a binomial trial 
$\mathrm{R}_{\mathrm{ij}} \sim \mathrm{B}(1,0.05)$. Noteworthy, that genetic recombination in the form of chromosomes swapping arms was not modeled in this study, and each gene could recombine individually (Fig. 2E). We assume that whenever the effects of genetic linkage are not studied (as is the case in the present work) such an assumption is valid. Of course, a natural crossing-over event swaps more genes at a time. However, since recombination frequency is arbitrary in our study, it's valid to assume that the specific mode of recombination is irrelevant on a per gene frequency basis as long as genetic linkage is not the focus of study. In the present study, another reason to implement individual gene recombination model was that we aimed, as part of the study, to explore the effects of the strength of genetic recombination per se on MR evolution, and the natural way crossing-over works imposes the confounding factor of genetic linkage. Should genetic linkage be addressed elsewhere using the present model, the code and algorithm for recombination should be adjusted accordingly to replicate the natural way of crossing-over.

In a similar way, using binomial trials, individuals that mate at a given step were determined based on a parameter that set a specific percent of the population that will mate. Individuals to

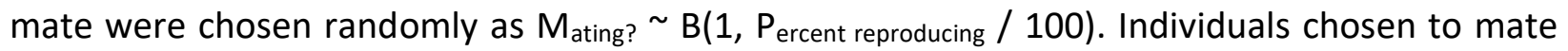
were randomly paired for mating.

During the mating process one progeny was produced per mating pair per mating event. Each parent passed on one of its chromosomes randomly at $50 \%$ probability (see equations in Fig. 2G).

As the final stage of each step, selection and individual survival was applied. The modeled population was assumed to inhabit an ecosystem that has a species-specific capacity of 10,000 individuals for the given modeled species. Therefore, at each step excess population that resulted from reproduction was removed stochastically, so that individuals of higher fitness were more likely to survive each simulation step. Relative fitness was determined by the relative proximity of a given individual to the current environmental optimum, which was simply the preset optimal $E$-value for the simulated adaptive trait in the case of stabilizing selection regimen or a moving adaptive trait $E$-values when directional selection was applied. For a detailed description and algorithm of how selection and survival was applied see Fig. 2, Supplement 1 (part "SURVIVAL") and Supplement 2.

Standard parameters were changed as needed for experimental purposes.

Statistical comparison of the obtained data was performed using the Matlab (MathWorks Inc, Massachusetts) implementation of standard statistical methods. Initial samples were checked for outliers, normality of distribution and further compared by relevant statistics indicated in the Results section for specific tests.

\section{Results}

\section{Model outline}

The general architecture of the underlying model is shown in Fig. 2. Model Matlab code is shown in Supplement 1. The algorithm for how selection was applied is shown in Supplement 2. 
bioRxiv preprint doi: https://doi.org/10.1101/2021.09 25.461822. this version posted September 26, 2021. The copyright holder for this preprint (which was not certified by peer review) is the author/funder, who has granted bioRxiv a license to display the preprint in perpetuity. It is made available under aCC-BY-NC-ND 4.0 International license.

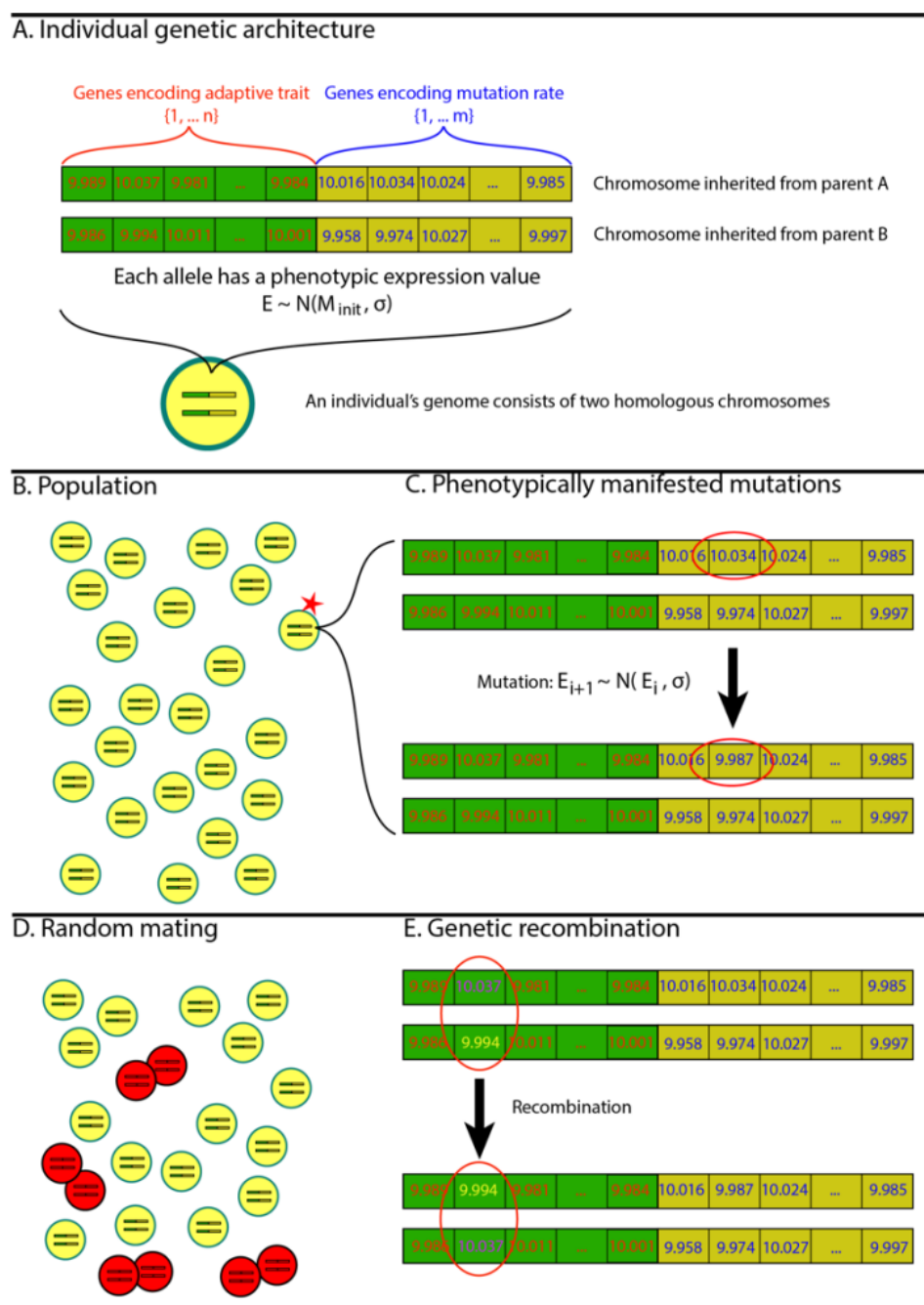

F. Reproduction G. Gamete segregation

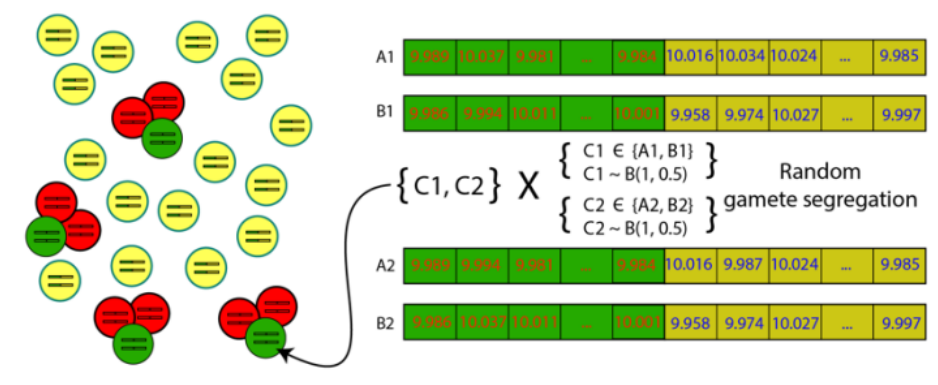

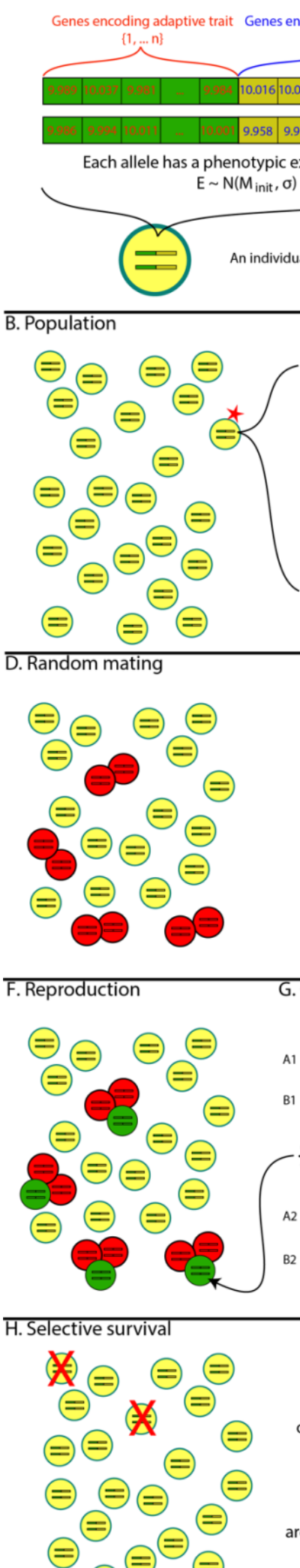

$$
\begin{aligned}
& \begin{array}{c}
\text { Gach allele has a phenotypic expring adaptive trait Genes en } \\
\end{array}
\end{aligned}
$$

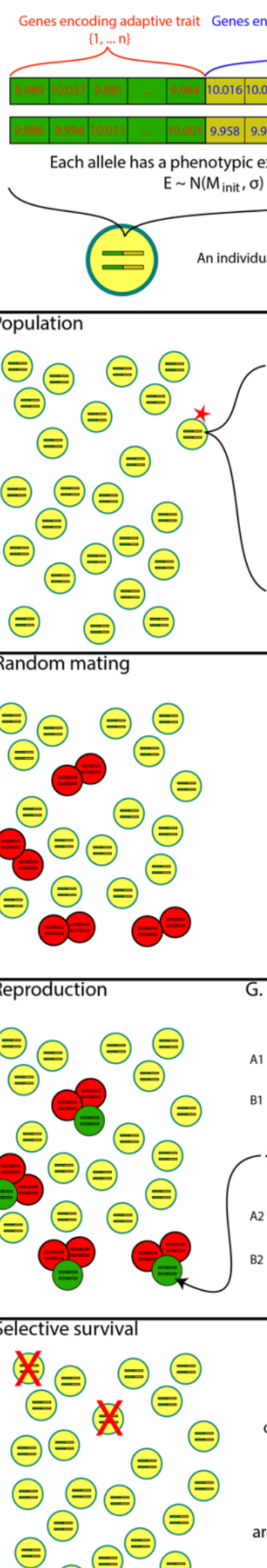

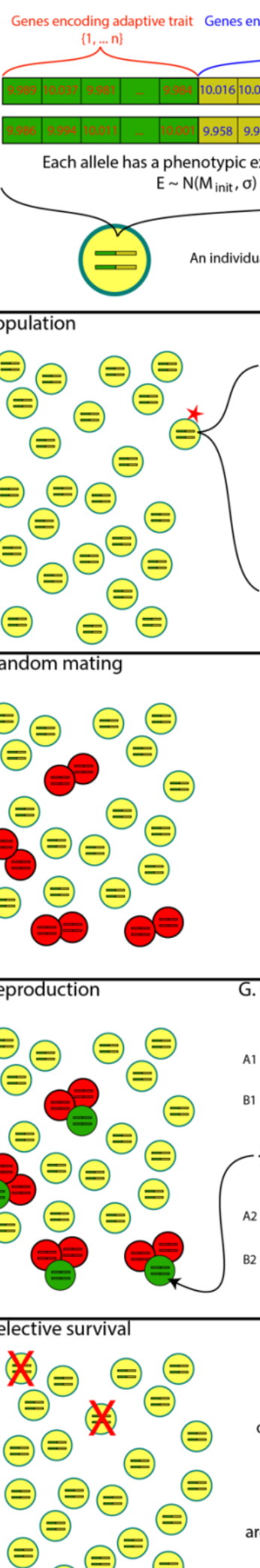


Fig. 2. Model algorithm for each simulation step. A, An individual's genome consists of two homologous chromosomes each inherited from one of the individual's parents. Each chromosome consists of two sets of genes: a) genes that encode adaptive phenotypic traits (left side in green) and b) those that encode MR (right side in yellow). Each gene has an expression level $(E)$ that in the initial population is generated from a normal distribution with the mean $\mathrm{M}$ (initial population optimum of 10 ) and a standard deviation representing variance of inheritance $\sigma(=$ variance of functional mutation effects). B, The population consists of sexually reproducing organisms and has a maximum population size imposed by the ecosystem's capacity. C, Functional mutations occur at the current individual's MR determined by the averaged expression of all alleles encoding MR multiplied by a fixed transformation coefficient common to all individuals. Functional mutations alter the expression value of the affected gene such that the gene's altered expression level is drawn from a normal distribution with the pre-mutation expression serving as the mean and a standard deviation $\sigma$. D, A subset of individuals (based on the reproduction frequency preset in the model) randomly mate for reproduction. E, Genetic recombination occurs at a present rate per gene per simulation step and is modeled as homologous alleles swapping their respective chromosomes. F, Each reproducing pair of individuals produce a single progeny. G, Gamete segregation is modeled by using Bernoulli trials such that each chromosome in each parent has a $50 \%$ chance of being transmitted to the progeny. $\mathbf{H}$, After reproduction, progeny are added to the population and selective elimination of individuals follows by applying Bernoulli trials for each individual such that an individual's chance of survival depends on the individual's fitness. Individual's fitness is determined by averaging the expression of all adaptive alleles and comparing it to the current environmental optimum (proximity means higher fitness). The overall frequencies of selective mortality are such that the remaining population approaches the ecosystem's capacity.

Selection acting on adaptive traits impacts the evolution of mutation rate.

We first proceeded with testing our overarching hypothesis that the mode of selection acting on an adaptive trait will impact the evolution of MR. As shown in Fig. 3A-B, under stabilizing selection the expression of the adaptive trait remains relatively constant and close to the initial population mean, while under directional selection it responds to selection toward increased expression. This measurement served as a control in order to ensure that the selection regimens indeed worked as expected in their effect on the adaptive trait. Another control, ensuring that the population numbers stayed at the imposed ecosystem's cap is shown in Fig. 3C-D, and confirms that the imposed population size cap worked as expected. Fig. 3E-F demonstrates that the initial distribution of MR did not differ between the stabilizing and direction selection setups (t-test; $p=0.49$; respective means equaled 10.004 and 9.993). Fig. 3G-H shows that at the end of the simulation the distribution of MRs significantly differs between selection regimens and provides support for our primary hypothesis that directional selection acting on adaptive traits alters the mode of evolution of MR (Wilcoxon rank sum test; $p<0.001$; means: 6.81 and 16.35). Wilcoxon ranks sum test will be used in testing all further results regarding the final MR. As we anticipated based on our model shown in Fig. 1, under stabilizing selection MR evolves toward lower values (mean=6.81), while directional selection leads to higher MRs (mean=16.35) in our experiment. Notably, directional selection creates initial transient drops in population size by, perhaps, imposing higher initial rates in mortality when the environment is changing and alters the fitness peak. During later stages of evolution, the population appears to catch up with the change and restores is cap size. This pattern will be observed throughout the remaining experiments. 
Stabilizng selection

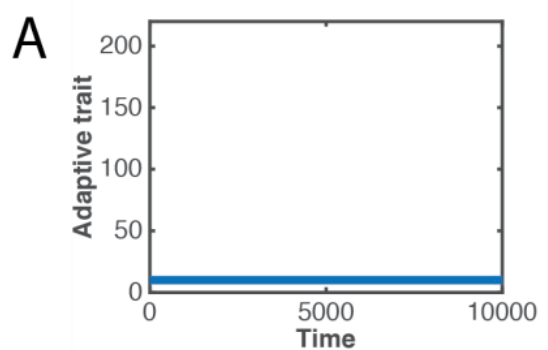

C

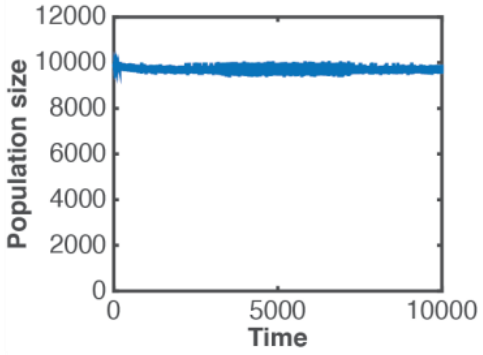

E

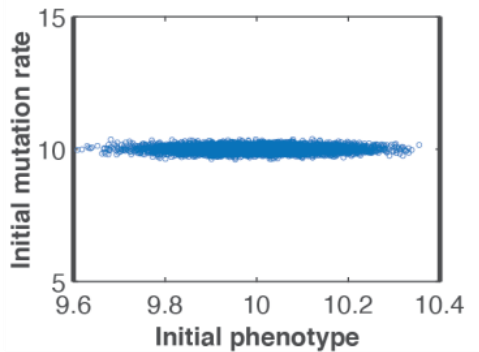

G
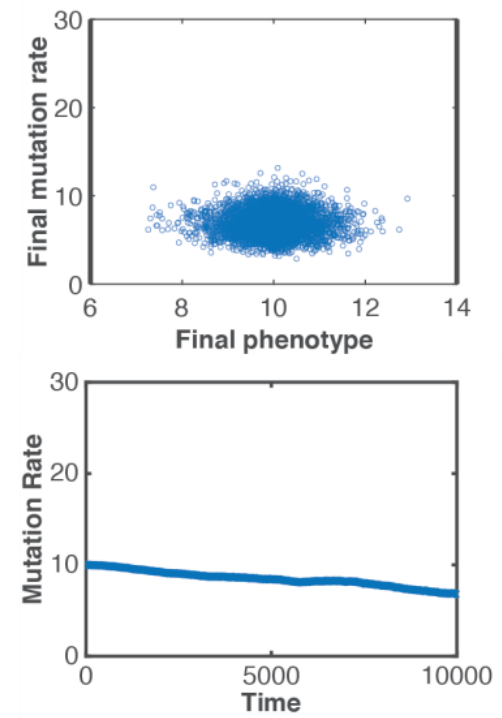

Directional selection

B

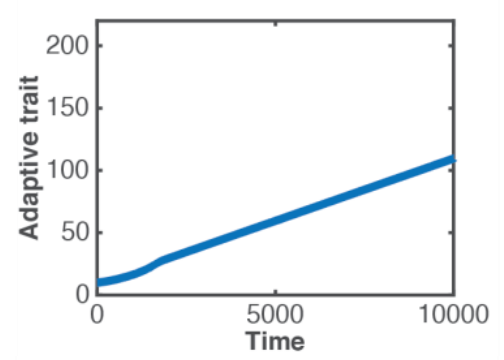

D

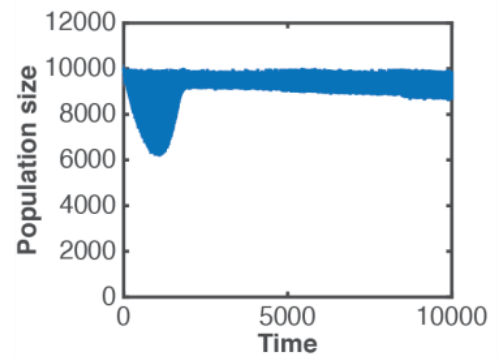

$\mathrm{F}$

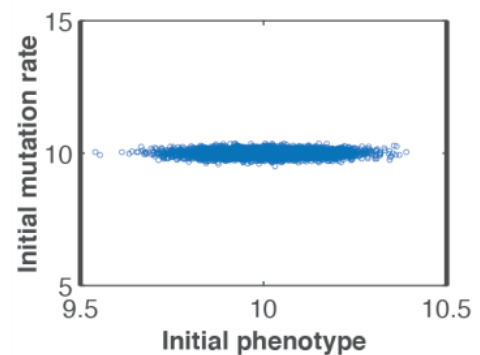

$\mathrm{H}$

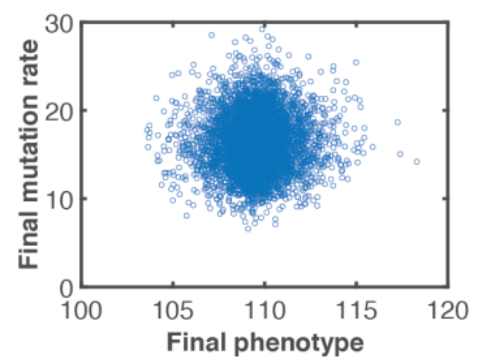

$J$

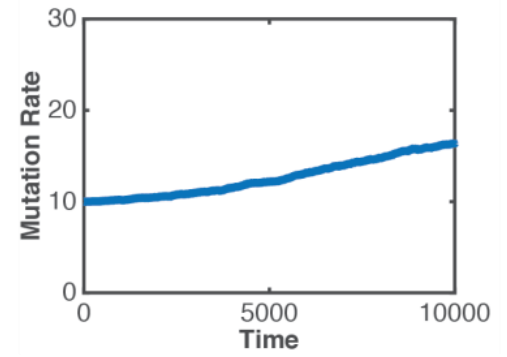

Fig. 3. The effect of selection acting on an adaptive trait on the evolution of mutation rate. Left panels represent evolutionary processes when the adaptive trait is under stabilizing selection. Right panels represent evolutionary processes when the adaptive trait is under directional selection toward increased expression. A-B, Adaptive trait expression (in arbitrary units) throughout the simulation time (in simulation updates). C-D, The dynamics of population (in the number of individuals) throughout the simulation time. E-F The distribution of MR (in arbitrary units) relative to the distribution of initial expression of the adaptive trait; each circle represents one individual. G-H, The distribution of MR relative to the distribution of adaptive trait expression at the end of the simulation. I-J, The evolution of the average MR in the population throughout the simulation time. 


\section{Stronger directional selection increases the observed differences in MR evolution.}

We further aimed to explore whether the regimen of selection acting on the adaptive trait was at least part of the causes that lead to the differences in MR evolution observed in Fig. 3G$\mathrm{H}, \mathrm{I}-\mathrm{G}$. We therefore increased the strength of directional selection by $30 \%$ relative to that applied in the experiment shown in Fig. 3. Henceforth, we will only show the distributions of MRs at the end of each simulation, while the dynamics of the control processes will be shown in supplementary figures. Increasing the strength of directional selection leads to a statistically significant increase in MR (Fig. 4A-B; $p<0.0001$; means: 16.35 and 20.85), providing an indication that directional selection acting on the adaptive trait is at least partially responsible for the observed differences in the evolution of MR. As expected, the adaptive trait (the X-axes in Fig. 4AB) also demonstrated a dramatic increase in expression in response to stronger directional selection. Population dynamics and other controls are shown in Supplement 4a (note a deeper initial drop in population size in response to a faster environmental change).

The effect of the strength of directional selection
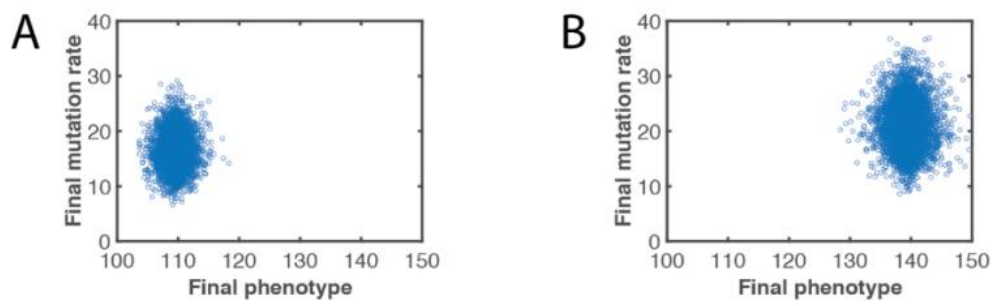

The effect of the cost of somatic mutations - stabilizing selection
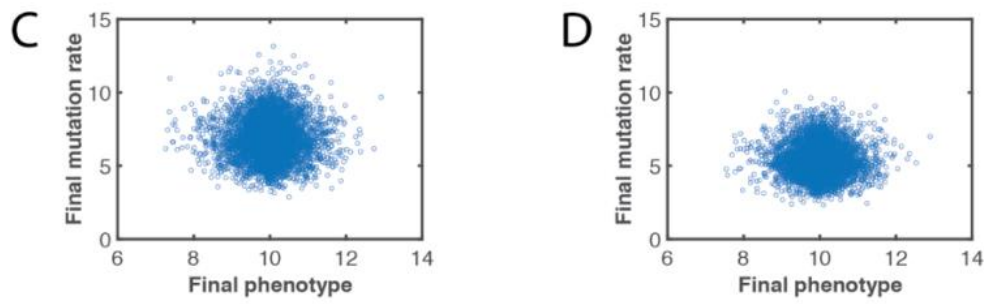

The effect of the cost of somatic mutations - directional selection

E

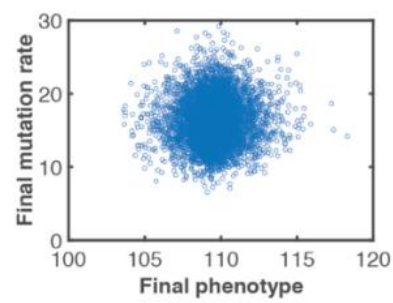

$\mathrm{F}$

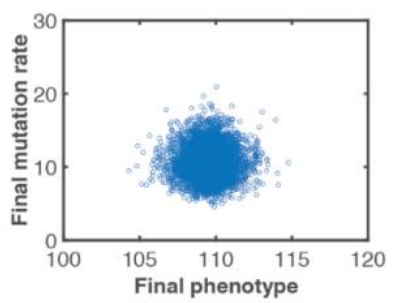

Fig. 4. The effect of the strength of directional selection and the cost of somatic mutations on MR evolution. A, MR evolution under the default (parameter value 0.001) strength of directional selection acting on the adaptive trait. B, MR evolution under increased (parameter value 0.0013 ) strength of directional selection acting on the adaptive trait. C, MR evolution with the default cost of somatic mutations (parameter value 0.001 ) and stabilizing selection acting on the adaptive trait. D, MR evolution with an elevated cost of somatic mutations (parameter value 0.1 ) and stabilizing selection acting on the adaptive trait. E, MR evolution with the default cost of somatic mutations 
(parameter value 0.001 ) and directional selection acting on the adaptive trait. F, MR evolution with an elevated cost of somatic mutations (parameter value 0.1 ) and directional selection acting on the adaptive trait.

The cost of somatic mutations alters MR evolution under both stabilizing and directional selection.

Theoretically, the evolution of MR shown above should be affected by the cost of somatic mutations, with the higher cost promoting selection for lower MR. In order to elucidate the role of the cost of somatic mutations, we increased the cost by 100 -fold under both stabilizing and directional selection acting on the adaptive trait. Under stabilizing selection acting on the adaptive trait, a higher cost of somatic mutations promoted stronger selection for lower MR, resulting in lower MR values (Fig. 4C-D; $p<0.001$; means: 6.81 and 5.4). Fig. 4E-F demonstrates that a higher cost of somatic mutations has a similar effect under directional selection by buffering the evolution of higher MR and leading to lower MR values at the end of simulation ( $p<0.001$; means: 16.35 and 10.6). Even with the lower MR, the final selected phenotype was similar for the two conditions. Control processes are shown in Supplements $4 \mathrm{~b}$ and $4 c$, correspondingly. Therefore, the combined results shown in Fig. 4C-F are consistent with the idea that the cost of somatic mutations is a modulator of MR evolution and a higher cost promotes selection for lower MR regardless of the regimen of selection acting on adaptive traits.

Multigenic inheritance of the adaptive trait has a significant impact on the evolution of mutation rate

If the hypothetical mechanism proposed in Fig. 1 is correct, we should also expect that selection acting on a monogenically inherited adaptive trait will result in a different pattern of MR evolution compared to a multigenic adaptive trait. A polygenic trait should require more mutations in order to change in response to selection. Therefore, under directional selection acting on a polygenic adaptive trait, we should expect a tendency toward a higher resulting MR, since selection will favor individuals that demonstrate a higher ability to deviate into the selected phenotypic tail. The ability of stabilizing selection acting on the adaptive trait to lower MR should, likewise, be buffered by multigenic inheritance of the adaptive trait, since a monogenic trait requires lower $M R$ to be changed in its phenotypic manifestation whereas selection acts to maintain proximity of the trait to the selected optimum. Data shown in Fig. 5 agree with such expectations. As can be seen in Fig. 5A, under stabilizing selection acting on a multigenic (10 genes) adaptive trait, evolution toward lower MR is observed (See also Fig.3). If the adaptive trait is monogenic (Fig. 5B), a significantly lower MR evolves ( $p<0.001$; means: 8.81 and 1.42), consistent with the idea that a monogenic trait requires fewer mutations to be altered, therefore selection acts to lower MR further. As expected for a monogenic trait, a much narrower distribution of phenotypes is observed (Fig. 5B). Multigenic traits require more mutations per the same amount of phenotypic change, and therefore higher MR is tolerated whereby the phenotypic expression of the adaptive trait remains stable. Controls are shown in Supplement 5a. Theoretically, if each mutation has less effect on the phenotype, we should see a reverse 
trend toward the higher MR under monogenic inheritance of the adaptive trait, since in such a scenario more mutations will have less effect on the trait. We made the inherited DPhE of mutations narrower (the parameter value was lowered from the standard 0.05 to 0.01 under stabilizing selection and 0.18 under directional; the latter value is higher, since 0.01 under all other parameters unchanged rendered the population non-responsive to directional selection by providing too little phenotypic variance and the population died out early in the simulation unable to adapt to the changing environment). Fig. 5C demonstrates that a narrower DPhE indeed restores higher values of the evolved MR (mean=8.23; all three experiments differ between each other with $p<0.0001$ ), consistent with the idea of a higher tolerance of a population to higher MR when mutations have smaller effects on the phenotype. Notice that, as expected, panels with lower MR demonstrate also lower phenotypic variance (X-axis; Fig.5A-C in standard deviations: $\sigma=0.567, \sigma=0.095, \sigma=0.18$ ). Fig. 5D-F demonstrates a very similar pattern under directional selection acting on the adaptive trait, with all the three experiments showing significant differences $(p<0.001)$ of the means and variances (controls for Fig. 5D-E are in Supplement 5b, for Fig. 5c,f in Supplements $5 c$ and 5d, respectively). As before, note that the final phenotype is similar despite differences in MR. The results shown in Fig. 5, therefore, corroborate the idea that the number of genes encoding adaptive traits that are under various regimens of selection significantly impacts MR evolution.

MULTIGENIC ADAPTIVE TRAIT

MONOGENIC ADAPTIVE TRAIT
MONOGENIC ADAPTIVE TRAIT NARROW DPhE

Stabilizing selection acting on adaptive trait

A

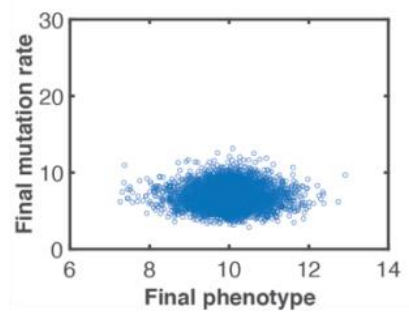

D

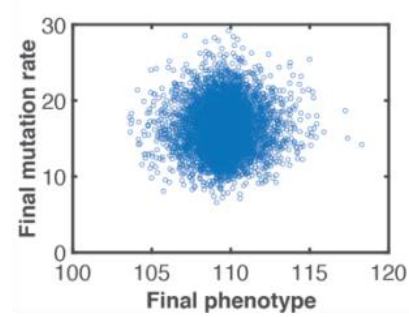

B

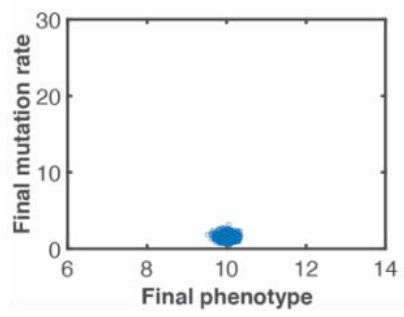

C

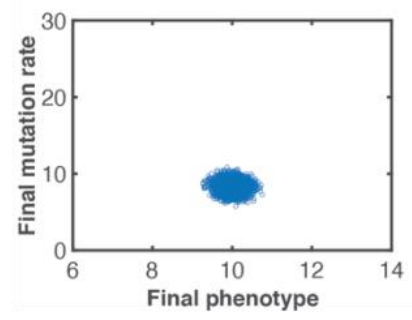

Directional selection acting on adaptive trait

E

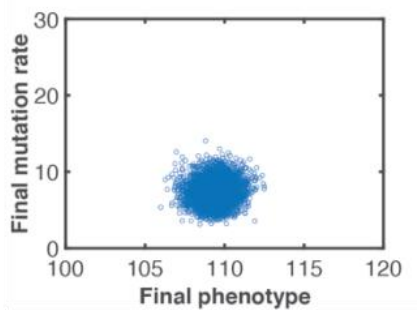

$\mathrm{F}$

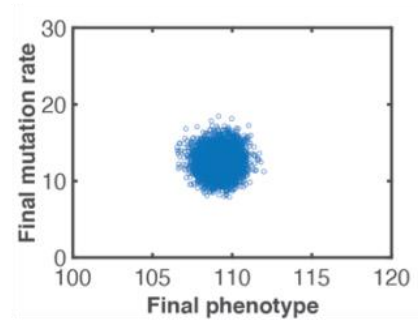

Fig. 5. The effect of multigenic inheritance of the adaptive trait on MR evolution. Left column: multigenic adaptive trait, middle column: monogenic adaptive trait, right column: monogenic adaptive trait with a narrow distribution of phenotypic effects (DPhE) of mutations. A-C, The effect of multigenic inheritance of the adaptive trait under stabilizing selection acting on the adaptive trait. D-F, The effect of multigenic inheritance of the adaptive trait under directional selection acting on the adaptive trait. 


\section{Multigenic inheritance of mutation rate buffers the effect of selection}

We further hypothesized that the number of genes encoding MR should have a profound effect on MR evolution. If theoretically MR was encoded by one gene, the gene would be better visible to selection given its direct association with the phenotypic expression of MR. Since selection acts on genes indirectly via phenotypic traits, multigenic inheritance should make each particular gene less visible to selection because each gene only partially contributes to the trait. As shown in Fig. 6A-B (controls in Supplement 6a), under stabilizing selection acting on the adaptive trait, selection is more efficient in lowering a monogenic MR compared to multigenic ( $p<0.001$; means: 6.81 for multigenic $M R$ and 1.26 for monogenic $M R$ ), revealing thus the hypothesized buffering effect of multigenic inheritance of MR on the ability of selection to lower MR under stabilizing selection. Fig. 6C-D (controls in Supplement 6b) also reveals a similar effect when the adaptive trait is under directional selection ( $p<0.001$; means: 16.35 and 4.12). The latter pattern can be explained by a direct visibility of the single gene underlying the MR trait to selection whereby the cost of MR triggers selection for a lower MR. In addition, for monogenically-encoded MR an allele that increases MR can easily be segregated away from the adaptive trait by recombination. Under multigenic encoding of MR, selection cannot act on each gene as strongly because each gene only has a partial contribution to the MR trait and higher MR alleles are accumulated in the population following the principle shown in Fig. 1.

MONOGENIC MR

MULTIGENIC MR

The adaptive trait is under stabilizing selection
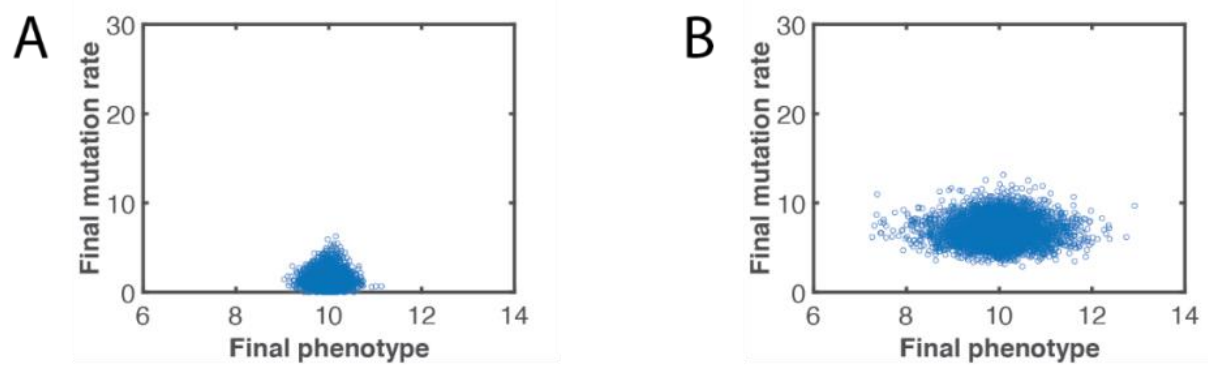

The adaptive trait is under directional selection
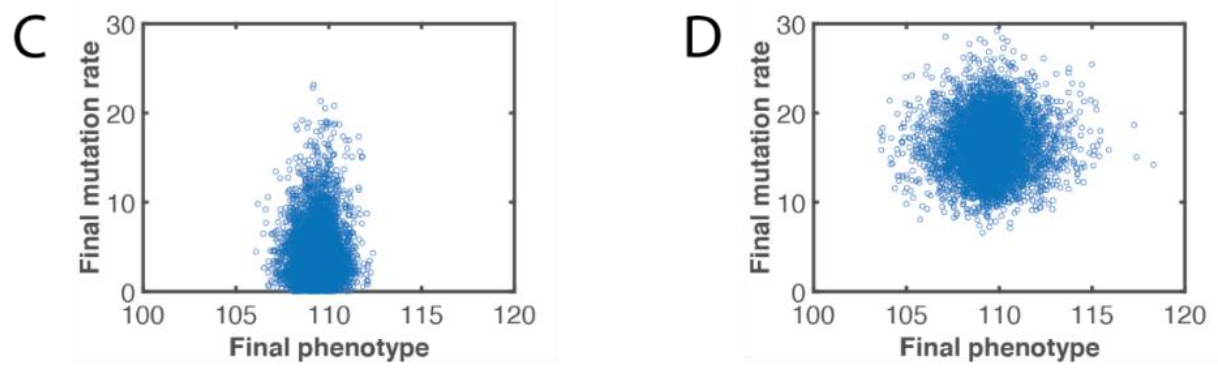

Fig. 6. The effect of multigenic inheritance of MR impacts its evolution. A, Evolution of multigenically (10 genes) encoded MR under stabilizing selection acting on the adaptive trait. B, Evolution of monogenically (1 gene) encoded MR under stabilizing selection acting on the adaptive trait. C, Evolution of multigenically (10 genes) encoded MR under directional selection acting on the adaptive trait. D, Evolution of monogenically (1 gene) encoded MR under directional selection acting on the adaptive trait. 
Genetic recombination buffers MR evolution under both stabilizing and directional selection acting on the adaptive trait

Clonally reproducing organisms, such as bacteria, have been shown to evolve higher MR when mutator alleles are genetically linked with traits providing higher fitness $(13,15)$. In sexually reproducing organisms, genetic recombination is capable of effectively separating mutator alleles from adaptive alleles, which is the primary reason it is believed that selection for higher MR is not possible in population with sexual reproduction. Our results presented so far indicate that selection for higher $M R$ is still possible even in the presence of genetic recombination. However, genetic recombination should be a factor which at least buffers selection acting on MR. We compared the evolution of MR in our simulated population in the standard setting versus a population with absent recombination, all other processes left intact (e.g. MR and the adaptive trait are each encoded by 10 genes). As can be seen in Fig. 7A-B (controls in Supplement 7a), genetic recombination significantly buffers selection in its ability to lower MR under stabilizing selection acting on the adaptive trait $(p<0.001$; means: 6.81 with and 2.78 without recombination). Comparison of Fig. 7A and Fig. 7B also reveals a significant contribution of genetic recombination to the phenotypic diversity evident from the much larger variance of the phenotypic expression of the adaptive trait in presence of genetic recombination, as expected. A similar buffering effect is visible under directional selection $(p<0.001$; means: 16.35 and 21.0), whereby recombination (Fig. 7C) obstructs the accumulation of high MR alleles in the population relative to the setting with no recombination (Fig. 7D). Controls for Fig. 7C-D in Supplement 7b. Therefore, our results demonstrate consistency with the general population genetics theory and indicate that genetic recombination is an important factor in MR evolution.

\section{RECOMBINATION PRESENT RECOMBINATION ABSENT}

The adaptive trait is under stabilizing selection
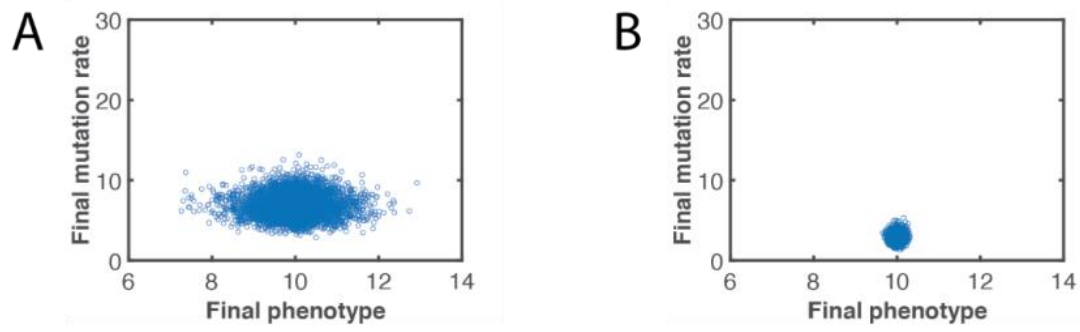

The adaptive trait is under directional selection
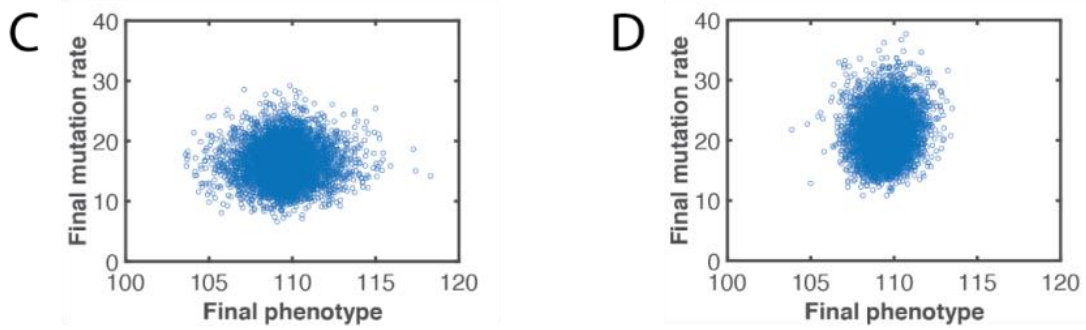

Fig. 7. The effect of genetic recombination on MR evolution. A, MR evolution in presence of genetic recombination under stabilizing selection acting on the adaptive trait. B, MR evolution in the absence of genetic 
bioRxiv preprint doi: https://doi.org/10.1101/2021.09.25.461822; this version posted September 26, 2021. The copyright holder for this preprint (which was not certified by peer review) is the author/funder, who has granted bioRxiv a license to display the preprint in perpetuity. It is made available under aCC-BY-NC-ND 4.0 International license.

recombination under stabilizing selection acting on the adaptive trait. C, MR evolution in presence of genetic recombination under directional selection acting on the adaptive trait. D, MR evolution in the absence of genetic recombination under directional selection acting on the adaptive trait.

Population size appears to have a limited effect on MR evolution in the range between 500 and 5,000,000 individuals

The effect of population size under stabilizing selection
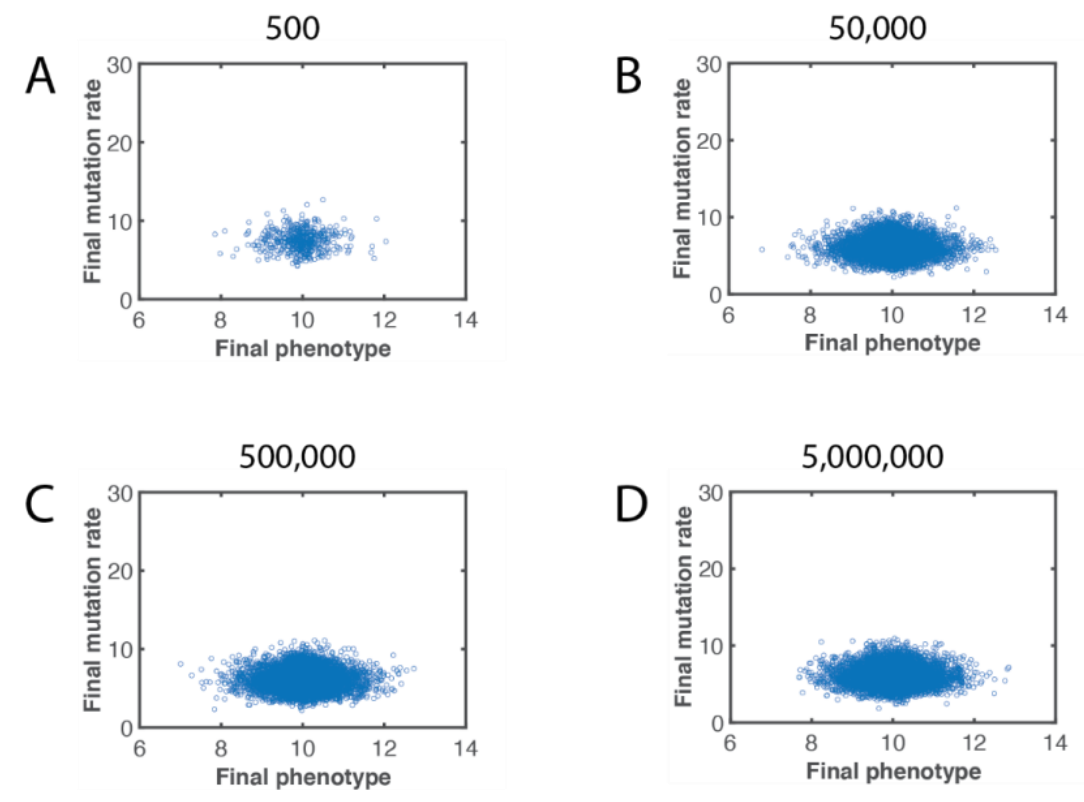

The effect of population size under directional selection
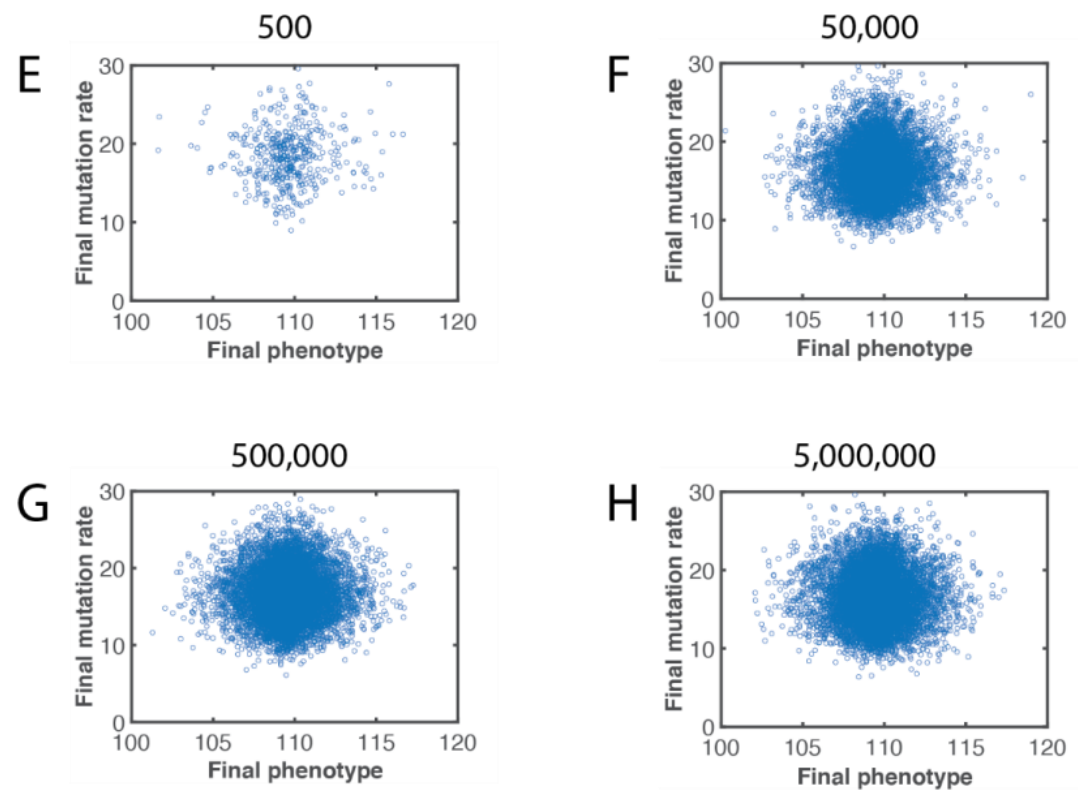

Fig. 8. The effect of population size on MR evolution. In all panels, numbers above charts indicate population size in individuals. 
As shown in Fig. 8, we tested the effect of population size on MR evolution with 4 population sizes of $500,50000,500000$, and 5000000 individuals, spanning overall 4 orders of magnitude, both under stabilizing (Fig. 8A-D; means: 7.47, 6.03, 6.05, 6.04) and directional (Fig. 8E-H; means: $18.41,16.49,16.6,16.55)$ selection acting on the adaptive trait. Although most pairwise comparisons in both settings result in p-value significantly below 0.001 , such a statistical difference becomes meaningless with such minimal differences among the means and such large sample sizes. Therefore, within the 4 orders of magnitude span of the tested population size and the other parameters we used in the model, we did not notice any profound effect of population size on MR evolution.

At least two possible explanations of such results can be proposed. First, it should be kept in mind that in modeling with arbitrary parameter values, only a positive result showing an effect can be interpreted as indicative of likely presence of such an effect in nature. A negative result showing no effect, on the other hand, can indicate that either such an effect is absent indeed or that the chosen parameter values do not provide the model with enough resolution to see an effect that exists in nature. Therefore, in the present case, our results showing little effect of population size on MR evolution might indicate that the model was not sensitive enough within the parameter values chosen.

However, we propose a different possible hypothetical mechanism that we deem likely to adequately describe the obtained results. Classical evolutionary synthesis operates with equations measuring the balance of selection and drift based on population size, e.g. (22-24), with an inherent assumption that the balance of drift and selection acts across the board for all individuals and is a constant property of a population of a given size and reproductive characteristics. Under such an assumption, the entire population should experience the same $\mathrm{drift} /$ selection ratio and the strength of selection should inversely depend on population size. Based on the statistical concept of "sampling error", there is reasonable doubt that population size does exert such an effect. However, a parameter missing from many such models is the relative distance in fitness between two alleles. In large populations, smaller differences between the relative fitness of two alleles will be visible to selection due to the highly population sizedependent nature of the strength of selection. Therefore, in a large population whose individuals show a continuous range of individual fitness, the fates of two alleles that are close to each other in fitness might still be discriminated by selection favoring the more fit. In small populations, on the other hand, where selection is weak, the fates of individuals of similar but slightly different fitness will more likely be determined by random processes. However, very large differences in the relative fitness of two exemplar alleles should make those alleles more visible to selection in terms of their probabilities to go to fixation or extinct. Based on this assumption, therefore, the absence of a tangible effect of population size on MR evolution obtained in our modeling can be explained by the "sampling bias" of selection shown in Fig. 1. In a large population, a substantial portion of the population is visible to both stabilizing and directional selection (Fig. 1A). In a small population (Fig. 1B), only a minor fraction of individuals is still visible to selection because of their sufficiently large fitness differential relative to the rest of the population. The same principle should hold for the least fit and most unfavored phenotypes, but those are not shown here for simplicity. In other words, if the balance of drift versus selection is not a uniform population sizedependent constant but forms a gradient instead (is distributed), the bias of selection to favor the most advanced expression of the selected trait will increase selection efficiency and thus 
counteract the general reduction in selection strength imposed by population size. As a result, the overall efficiency of selection to drive adaptation will depend on the balance of two oppositely counteracting factors - the general strength of selection inversely dependent on population size versus selection "sampling bias" directly dependent on population size, with the two being able to largely cancel each other out. In Fig. 1A, for example, high strength directional selection samples phenotypes ranging from high to modest MR. When population is small (Fig. 1B), only high MR phenotypes are still under now weaker selection, making the average MR in the selected part of the population higher compared to a large population shown in panel A. The resulting evolution of MR becomes in this way similarly paced in both cases. Noteworthy, Fig. 1 is a gross simplification of the proposed principle, since for a continuously distributed quantitative trait, as MR could be, the balance of drift versus selection should also be continuously distributed, not defined as in Fig. 1.

\section{Discussion}

Our results corroborate our initial hypothesis that the evolution of MRs in sexually reproducing populations is not universally directed toward lower MRs but is instead dependent on the general regimen of selection acting on adaptive traits. As shown in Fig. 1, stabilizing selection favoring adaptive trait expression closer to the population mean acts over generations to enrich the population with individuals harboring alleles that provide a lower MR, since such individuals tend to deviate less in their expression of the adaptive trait from the favored optimum. However, when directional selection is applied, favoring phenotypically deviant individuals from the tails of the phenotypic distribution of the adaptive trait, preferential survival is characteristic for individuals harboring alleles for a higher $M R$, since such individuals tend to deviate in the expression of their adaptive trait further from the population mean. As a result (Fig. 3), we observe a reversal of the directionality of MR evolution. While we cannot speculate based solely on a model on the absolute strength of directional selection to reverse the direction of MR evolution, our results still indicate that directional selection acting on adaptive traits at least buffers the putative universal selection toward lower MRs. Evidence that selection regimen acting on adaptive traits has at least some role in modulating the evolution of MRs is further elucidated when we applied stronger directional selection (Fig. 4A-B) and observed that its effect on MR evolution was further magnified.

As proposed by others $(1,9-12)$, the reason selection acts to lower MR is in the fitness cost of mutations. The cost of MR should be manifested both in the increased phenotypic variance of offspring (with more offspring deviating from optimal trait values) and in the costs of concomitantly altered somatic MR (as germline and somatic MRs are linked, and mutations can contribute to aging, cancer and other fitness-limiting impairments). This conjecture is corroborated by our results (Fig. 4C-F), whereby increasing the fitness cost of mutations leads to lower resulting MRs under both stabilizing and directional selection acting on the adaptive trait relative to their respective controls under stabilizing and directional selection. Therefore, a higher cost of mutations buffers the ability of directional selection to promote higher MRs and further increases the ability of stabilizing selection regimen to lower MR. This result implies that the actual ability of the general selection regimen acting on adaptive traits to modulate MR evolution will depend on the relative strength of selection compared to the fitness cost of 
mutations. Our previous modeling indicated that one mechanism to buffer the cost of somatic mutations is greater investment in somatic tissue maintenance, allowing for the evolution of higher MR and thus facilitating directional selection (25).

A substantial portion of phenotypic traits, including MR, are known to be encoded by multiple genes. For MR, these include the myriad of genes involved in DNA replication and repair, as well as metabolic and stress response genes that modulate the chemical and biochemical intracellular environment (see Introduction). Our results reveal that the multigenic nature of both adaptive traits and MR has a profound effect on MR evolution. As we demonstrate in Fig. 5, multigenic inheritance of the adaptive trait buffers the ability of stabilizing selection to lower MR. This pattern can be explained by the lower number of mutations needed to alter a monogenic adaptive trait (just one gene to change), so that individuals that are closer in their phenotypic expression of a monogenic adaptive trait should harbor alleles providing a much lower resulting MR. If the adaptive trait is multigenic (and we assume for simplicity roughly equal impact of each gene on the trait), deviation of such a trait from the selected population mean will require more mutations, and thus a higher MR is "tolerated" by stabilizing selection. Under directional selection, following the same logic, a multigenic adaptive trait leads to the evolution of a significantly higher MR compared to a monogenic trait, since more mutations are needed to alter a multigenic adaptive trait and therefore individuals that significantly deviate from the population mean phenotype (and favored by directional selection) are likely to harbor more alleles encoding a higher MR and/or alleles that have a more profound effect on MR. These results are also consistent with the mechanism proposed in Fig. 1 and our initial hypothesis.

We further demonstrate (Fig. 6) that multigenic inheritance of MR also has a notable effect on MR evolution. Stabilizing selection is more efficient in lowering monogenically inherited MR. This pattern can be explained by the fact that selection does not directly act on genes, but rather it acts on phenotypic traits (a point often ignored since the early 1900s - but perforce the original Darwinian view). For monogenic traits the encoding gene is directly visible to selection. While monogenic traits, such as mismatch repair deficiency caused by a single inactivating gene mutation (whether in yeast or humans), have been well described, it is likely that most variation in $M R$ in a population cannot be ascribed to variation in a single gene $(16,18,26)$. Most population variability of $M R$ in humans shows relatively moderate variation $(18,19)$, although some extremes are indeed infrequently observed in the human population. There should exist strong selection against such monogenic mutations in natural populations. Modest changes in MR may confer a minimal cost - while selection may still be able to act on the overall MR phenotype, the strength of selection acting on alleles of any particular gene contributing to MR (if highly multigenic) will be very weak or negligible. Together with co-selection for higher MR with deviant phenotypes under selection at the tails of a phenotypic distribution, this reasoning can provide a solution to the dilemma for how higher MR can be co-selected despite its cost and given that evolution has "no eyes for the future".

In multigenic inheritance, the contribution of each single gene (assuming all genes have effects of comparable scale) to the resulting phenotype is smaller and can be compensated by other encoding genes. Therefore, stabilizing selection is less efficient at purging each individual unfavorable allele if MR is multigenic. We also observe (Fig. 6C-D) that directional selection acting on the adaptive trait promotes a higher MR, if the latter is encoded by multiple genes. This can be explained following the same principle that the contribution of each individual MR-related 
gene to the lower fitness of a phenotype (due to the somatic and germline costs of mutations) having a higher net MR is less visible to selection compared to a MR encoded by a single gene. These results further corroborate our initial hypothesis illustrated in Fig. 1.

The main reason why natural selection is generally thought to universally act to lower MR in sexually reproducing populations is perhaps the fact that part of the sexual process is genetic recombination. The latter introduces shuffling of genes in their position on chromosomes relative to other genes, thus effectively disrupting genetic linkage. Such shuffling prevents many genes from being co-selected whereby one adaptive allele is selected for and others are co-selected by being physically located within the same genetic structure (such as for example a chromosome or a bacterial genome). Given that mutations have a supposed negative net effect on fitness due to the cost of somatic mutations, higher MRs are an unfavorable trait. We therefore further hypothesized that genetic recombination should buffer the effects of selection regimens on MR evolution, both under stabilizing and directional selection acting on adaptive traits. Our results (Fig. 7) demonstrate indeed that the presence of genetic recombination buffers the ability of stabilizing selection to lower MR, as well as the ability of directional selection to promote the evolution of higher MRs, obviously because of the above-mentioned selection-independent randomization of the genetic location of alleles relative to each other. We therefore should make a reservation here that the effects of selection regimen on the evolution of MRs observed so far will depend on the actual impact of genetic recombination relative to the strength of selection in a real natural population, which probably varies among populations and evolutionary circumstances.

We finally tested the hypothesis proposed earlier by Lynch (1) that population size, which is thought to determine the relative strength of selection and random drift in populations, should impact MR evolution. Contrary to conventional expectation, our results show no significant effect of population size on MR evolution regardless of the selection regimen. One possible explanation of such results can be the reservation that we have mentioned earlier that the absence of an effect in modeling with arbitrary parameter values can potentially be caused by choosing parameter values that are out of their effective natural range. However, an alternative explanation that we propose in this study is that the balance of drift and selection is not a uniform constant property of any given population based on its size but is rather a distributed phenomenon whereby part of the population remains under a greater influence of selection even when population size is reduced. The "sampling bias" principle shown in Fig. 1 should counteract the weakening of selection imposed by small population size and thus improve the ability of a population to evolve. Such a hypothesis appears to contradict the modern convention that describes the relative strength of drift and selection to be based on the statistical "sampling error" concept introduced by population size. However, the efficiency of selection is also determined by selection coefficient (27), which essentially is a measure of fitness differential between two alleles. Higher fitness differential imposes more efficient selection on the advantageous allele. In large populations, where selection is generally stronger, relatively small differences in fitness between two alleles can lead to non-random selection-directed increase in the frequency of the more beneficial allele relative to its less fit counterpart. When population size is reduced, the fates of such alleles become more random, drift driven. However, if fitness differential between those alleles increased (theoretically), equivalent strength of selection determining the relative fates of those alleles could be maintained in a smaller population. This 
implies that selection strength-wise, reduction in population size can be compensated by a larger difference in fitness (selection coefficient). Fig. 1 demonstrates this principle in the form of a "sampling bias" whereby in a population of reduced size, extremely adaptive phenotypes are still visible to selection; by "sampling" only a small subset of the most advanced phenotypes selection enhances the rate of evolution and compensates the population size imposed higher presence of drift.

Overall, our study reveals a rather complex pattern of factors that likely impact MR evolution in sexually reproducing populations and raises a number of questions that warrant further investigation. For example, in real populations many traits, like house-keeping physiological functions, are perhaps almost always under stabilizing selection, even when directional selection acts on some other trait (or one trait). It is therefore unclear whether directional selection acting on one trait will be enough to promote the evolution of higher MRs, as stabilizing selection acting on other traits might buffer this effect depending on the relative cost of higher MR in relation to different traits in a complex phenotype. Intuitively, however, we can speculate that when one or some traits come under strong directional selection, the value of such traits is high from the standpoint of adaptation and survival and thus the negative effect of a higher MR on other traits might be better tolerated. If the effect of reversal of selection to favoring higher MR shown in our study is real in natural populations, it is interesting whether such a process could contribute to the evolution of large organisms with small populations during the process of rapid evolutionary transitions $(28,29)$. Given that small populations are less responsive to selection, rapidly evolving a higher MR could enhance their evolvability when strong directional selection acts on such populations. Evidence of such processes already exists, as it has been shown, for example, that MR has recently undergone a rapid evolution in humans (18). Adaptive change in the speciation process can also be quite rapid, often interrupting longer periods of relative stability of entire species (30). Specific models involving rapid evolution in conjunction with speciation include "punctuated equilibria" $(28,29)$ and Vrba's cross-genealogical Turnover Pulse Hypothesis (31), involving patterns of extinction, survival and speciation triggered by environmental events within regional ecosystems. Theoretical explorations of events of even larger-scale morphological change in evolution, such as Simpson's Quantum Evolution (32) and Goldschmidt's Macroevolution (33), invoked various genetic parameters as known and understood ca. mid-20 ${ }^{\text {th }}$ century. Such formulations might well be re-evaluated given our results. Perhaps, as well, a pathway for a better integration of the theoretical postulates of evo-devo with conventional selectionist evolutionary theory might also be enabled through a consideration of our results.

It is also unclear whether lifespan can be a factor in MR evolution (25), as perhaps the cost of somatic mutations might be lower for short-lived species, which could potentially amplify the process of MR evolution reversal under directional selection regimens shown in the present study. Indeed, recent evidence indicates that somatic MR scales inversely with lifespan across mammals (34).

Among other questions, theoretically it is unclear how the process we have revealed by modeling can be classified in evolutionary theory. Under a stabilizing selection regimen lowering of MR appears to be the result of direct selection acting on MR due to the negative effects of mutations on fitness. However, the reversal towards evolving higher MRs that we show cannot be a result of selection, since selection will not directly promote a trait that has a negative effect 
on fitness (that would contradict the general theory of selection). Referring back to Fig. 1, we can speculate that this process, if real, should be rather unique in nature and may only apply to MR and no other traits, since MR is critical in generating diversity in all traits. Given the traditional supposition in population genetics (hence evolutionary theory in general) that rate and nature of mutations are inherently decoupled from the variation needed for directional selective (adaptive) change to occur, the postulate that MR could actually increase under directional selection on phenotypic traits in time of environmental change or stress appears rather controversial. Variation is not supposed to arise "on demand" - a teleological, even neoLamarckian position. However, our hypothesis merely sees positive selection on the tails of phenotypic distribution bringing along higher MR in genes underlying newly selected-for traits simply as an accidental byproduct of the change of selective regime itself - a coincidental sideeffect, rather than a purposive linkage between mutation and selection.

And finally, it should be noted that our modeling, being generalistic by nature, has some inherent limitations. For example, directional selection acting on monogenic non-quantitative traits will likely have little to no effect on MR evolution, since those traits do not demonstrate a continuous distribution of phenotypic expression. Also, real parameter values of the sexual process in many different natural populations and evolutionary scenarios will vary, therefore we can expect that in certain specific cases of MR evolution the main underlying factors may differ from those we revealed in our study. Nonetheless, our study raises a number of fundamental novel questions in regard to MR evolution and population size-related evolutionary phenomena and should instigate more specific studies, including experimental tests of the proposed processes.

\section{Acknowledgements}

These studies were supported by grants from the Veteran's Administration (1 101 BX004495) and the National Institute of Aging (R01AG066544 and R01AG067584) to J.D. We thank John Thompson of the University of California Santa Cruz for insightful suggestions on this work. 


\section{References}

1. M. Lynch, Evolution of the mutation rate. Trends Genet. 26, 345-352 (2010).

2. J. W. Drake, A constant rate of spontaneous mutation in DNA-based microbes. Proc. Natl. Acad. Sci. U. S. A. 88, 7160-7164 (1991).

3. K. J. Dawson, Evolutionarily stable mutation rates. J. Theor. Biol. 194, 143-157 (1998).

4. J. W. Drake, B. Charlesworth, D. Charlesworth, J. F. Crow, Rates of spontaneous mutation. Genetics 148, 1667-1686 (1998).

5. C. D. Laird, B. L. McConaughy, B. J. McCarthy, Rate of fixation of nucleotide substitutions in evolution. Nature 224, 149-154 (1969).

6. A. P. Martin, S. R. Palumbi, Body size, metabolic rate, generation time, and the molecular clock. Proc. Natl. Acad. Sci. U. S. A. 90, 4087-4091 (1993).

7. M. Lynch, et al., Genetic drift, selection and the evolution of the mutation rate. Nat. Rev. Genet. 17, 704-714 (2016).

8. W. Sung, M. S. Ackerman, S. F. Miller, T. G. Doak, M. Lynch, Drift-barrier hypothesis and mutation-rate evolution. Proc. Natl. Acad. Sci. U. S. A. 109, 18488-18492 (2012).

9. M. Lynch, The cellular, developmental and population-genetic determinants of mutationrate evolution. Genetics 180, 933-943 (2008).

10. A. H. Sturtevant, Essays on Evolution. I. On the Effects of Selection on Mutation Rate. $Q$. Rev. Biol. 12, 464-467 (1937).

11. E. G. Leigh, Natural Selection and Mutability. Am. Nat. 104, 301-305 (1970).

12. T. Johnson, Beneficial mutations, hitchhiking and the evolution of mutation rates in sexual populations. Genetics 151, 1621-1631 (1999).

13. C. F. Gentile, S. C. Yu, S. A. Serrano, P. J. Gerrish, P. D. Sniegowski, Competition between high-and higher-mutating strains of Escherichia coli. Biol. Lett. 7, 422-424 (2011).

14. L. Chao, E. C. Cox, Competition Between High and Low Mutating Strains of Escherichia coli. Evolution (N. Y). 37, 125 (1983).

15. P. D. Sniegowski, P. J. Gerrish, R. E. Lenski, Evolution of high mutation rates in experimental populations of E. coli. Nature 387, 703-705 (1997).

16. J. Pothof, et al., Identification of genes that protect the $\mathrm{C}$. elegans genome against mutations by genome-wide RNAi. Genes Dev. 17, 443-448 (2003).

17. D. Galetzka, et al., Expression of somatic DNA repair genes in human testes. J. Cell. Biochem. 100, 1232-1239 (2007).

18. K. Harris, J. K. Pritchard, Rapid evolution of the human mutation spectrum. Elife 6 (2017).

19. D. F. Conrad, et al., Variation in genome-wide mutation rates within and between human families. Nat Genet 43, 712-714 (2011).

20. L. Ségurel, M. J. Wyman, M. Przeworski, Determinants of mutation rate variation in the human germline. Annu. Rev. Genomics Hum. Genet. 15, 47-70 (2014).

21. V. E. Shelford, Some Concepts of Bioecology. Ecology 12, 455-467 (1931).

22. S. Wright, EVOLUTION IN MENDELIAN POPULATIONS. Genetics 16, 97-159 (1931).

23. R. A. Fischer, The genetical theory of natural selection (Clarendon Press, 1930).

24. P. A. P. Moran, Random processes in genetics. Math. Proc. Cambridge Philos. Soc. 54, 6071 (1958).

25. A. Rozhok, J. Degregori, Somatic maintenance impacts the evolution of mutation rate. 
BMC Evol. Biol. 19, 172 (2019).

26. E. A. Boyle, Y. I. Li, J. K. Pritchard, An Expanded View of Complex Traits: From Polygenic to Omnigenic. Cell 169, 1177-1186 (2017).

27. J. H. Gillespie, Population genetics : a concise guide. 214 (2004).

28. N. Eldredge, The Allopatric Model and Phylogeny in Paleozoic Invertebrates. Evolution (N. Y). 25, 156-167 (1971).

29. N. Eldredge, S. J. Gould, Punctuated equilibria: an alternative to phyletic gradualism. Model. Paleobiol., 82-115 (1972).

30. N. Eldredge, et al., The dynamics of evolutionary stasis. Paleobiology 31, 133-145 (2005).

31. E. S. Vrba, Environment and evolution: alternative causes of the temporal distribution of evolutionary events. Palaeoclim. Evol. I. Proc. Work. Palisades, NY, 1984 81, 229-236 (1985).

32. G. Simpson, Tempo and mode in evolution (Columbia University Press, 1944).

33. R. Goldschmidt, The material basis of evolution (Yale University Press, 1982).

34. A. Cagan, et al., Somatic mutation rates scale with lifespan across mammals. bioRxiv, 2021.08.19.456982 (2021). 